\title{
ORIGINAL ARTICLE \\ Evolution of apomixis loci in Pilosella and Hieracium (Asteraceae) inferred from the conservation of apomixis-linked markers in natural and experimental populations
}

\author{
ML Hand ${ }^{1}$, P Vít ${ }^{2}$, A Krahulcová ${ }^{2}$, SD Johnson ${ }^{1}$, K Oelkers ${ }^{1}$, H Siddons ${ }^{1}$, J Chrtek Jr ${ }^{2,3}$, \\ $\mathrm{J} \mathrm{Fehrer}^{2}$ and AMG Koltunow ${ }^{1}$
}

The Hieracium and Pilosella (Lactuceae, Asteraceae) genera of closely related hawkweeds contain species with two different modes of gametophytic apomixis (asexual seed formation). Both genera contain polyploid species, and in wild populations, sexual and apomictic species co-exist. Apomixis is known to co-exist with sexuality in apomictic Pilosella individuals, however, apomictic Hieracium have been regarded as obligate apomicts. Here, a developmental analysis of apomixis within 16 Hieracium species revealed meiosis and megaspore tetrad formation in 1 to $7 \%$ of ovules, for the first time indicating residual sexuality in this genus. Molecular markers linked to the two independent, dominant loci LOSS OF APOMEIOSIS (LOA) and LOSS OF PARTHENOGENESIS (LOP) controlling apomixis in Pilosella piloselloides subsp. praealta were screened across 20 phenotyped Hieracium individuals from natural populations, and 65 phenotyped Pilosella individuals from natural and experimental cross populations, to examine their conservation, inheritance and association with reproductive modes. All of the tested $L O A$ and LOP-linked markers were absent in the 20 Hieracium samples irrespective of their reproductive mode. Within Pilosella, LOA and LOP-linked markers were essentially absent within the sexual plants, although they were not conserved in all apomictic individuals. Both loci appeared to be inherited independently, and evidence for additional genetic factors influencing quantitative expression of $\angle O A$ and $\angle O P$ was obtained. Collectively, these data suggest independent evolution of apomixis in Hieracium and Pilosella and are discussed with respect to current knowledge of the evolution of apomixis.

Heredity (2015) 114, 17-26; doi:10.1038/hdy.2014.61; published online 16 July 2014

\section{INTRODUCTION}

The process of apomixis (asexual seed production) has been studied in various plant species, not only for its potential value to agricultural plant breeding, but also for its intriguing role in plant evolution. Apomixis is developmentally variant and has evolved independently many times across different angiosperm plant families (Carman, 1997). Although sexual seed formation generates variation in progeny through meiosis and fertilization, gametophytic apomictic processes produce chromosomally unreduced eggs in female gametophytes (or embryo sacs) through the avoidance of meiosis (apomeiosis). Embryos develop from the unreduced eggs in the absence of fertilization (termed parthenogenesis) and endosperm formation occurs with or without fertilization. The progeny resulting from apomictic reproduction always retain a maternal genotype (Bicknell and Koltunow, 2004; Ozias-Akins, 2006).

Gametophytic apomixis is separated into two types: apospory and diplospory, depending on the type of cell that gives rise to the chromosomally unreduced embryo sac. In diplospory, the diploid megaspore mother cell (MMC), which usually undergoes meiosis in sexual reproduction, develops into an embryo sac in the absence of meiosis (Tucker and Koltunow, 2009). During apospory, an unreduced embryo sac develops from an ovule cell, called an aposporous initial (AI) cell, positioned adjacent to sexual embryo sac precursors. In both forms of gametophytic apomixis, embryo development is fertilization independent via parthenogenesis, while endosperm formation may or may not require fertilization (Koltunow et al., 2013).

The Hieracium and Pilosella genera of hawkweeds (previously treated as Hieracium subgenus Pilosella) contain closely related species of Eurasian origin, which have evolved via numerous hybridization and polyploidization events. Both genera contain mostly polyploid species and few diploid species (Fehrer et al., 2007a; Krak et al., 2013). Sexual and apomictic species are found in both genera (albeit at different frequencies), but the route taken to form chromosomally unreduced embryo sacs is diplosporous in Hieracium and aposporous in Pilosella. In both apomictic Hieracium and Pilosella, embryo and endosperm formation are fertilization independent. To date, apomictic Hieracium species have been considered near-obligate apomicts, appearing to produce only clonal maternal progeny.

${ }^{1}$ Commonwealth Scientific and Industrial Research Organization (CSIRO) Plant Industry, Waite Campus, Glen Osmond, SA, Australia; ${ }^{2}$ Institute of Botany, Academy of Sciences of the Czech Republic, Zámek 1, Průhonice, Czech Republic and ${ }^{3}$ Department of Botany, Faculty of Science, Charles University in Prague, Benátská 2, Prague, Czech Republic

Correspondence: Dr AMG Koltunow, Commonwealth Scientific and Industrial Research Organization (CSIRO) Plant Industry, Waite Campus, PO Box 350, Glen Osmond, SA 5064, Australia.

E-mail: Anna.Koltunow@csiro.au

Received 28 January 2014; revised 30 May 2014; accepted 3 June 2014; published online 16 July 2014 
However, apomixis has not been examined extensively in Hieracium species, having been documented in 12 species by Bergman (1941), and in a single species (H. alpinum L.) by Skawińska (1963). Some polyploid Hieracium are pollen fertile but abnormal pollen development has also been reported, resulting in male sterile plants (Mráz et al., 2005; Slade and Rich, 2007). Hieracium experimental hybrids also set very few seed or are completely seed sterile (Mráz and Paule, 2006). As a result, Hieracium species have not been a major focus for genetic analyses of apomixis.

By contrast, Pilosella has been developed as a model system for the analysis of apomixis occurring by aposporous gametophyte formation coupled with fertilization-independent seed formation. A suite of characterized accessions, mutants and genetic and molecular tools have been developed to identify causal genes (Bicknell and Koltunow, 2004; Koltunow et al., 2013). Unlike Hieracium, inter-cytotype and inter-specific crosses are common in nature for Pilosella, and experimental crosses confirm that reproductive barriers are almost completely absent (for example, Fehrer et al., 2005, 2007b; Okada et al., 2011).

Pilosella apomicts are facultative apomicts meaning that they retain a capacity to form a percentage of progeny via the sexual pathway. This results in three additional types of non-apomictic progeny termed 'off-type' or 'variable'. The nomenclature of Harlan and deWet (1975) designates the dominant apomictic progeny as $2 n+0$ in relation to female and male gamete ploidy and parental contribution, respectively. Progeny also result from the fertilization-independent development of an embryo from a meiotically reduced egg and are designated as $n+0$ individuals, in addition to sexual progeny designated as $n+n$. The chromosomally unreduced eggs that develop into aposporous embryo sacs can also be fertilized to produce progeny of increased ploidy designated as $2 n+n$ (Bicknell et al., 2003). Facultative apomictic Pilosella species produce meiotically reduced fertile pollen and can act as both male and female parents in hybridizations (Fehrer et al., 2007b). Therefore, if two or more Pilosella species/cytotypes occur in a population, they can form hybrid swarms in which the parental biotypes, their hybrids and the products of multiple hybridizations (including backcrosses) produce complicated population structures containing plants of differing ploidy levels (Krahulcová et al., 2009).

Taxonomy of Hieracium and Pilosella is complex and the classifications of Nägeli and Peter (1885) and Zahn (1921-1923) are used to define basic and intermediate species. According to this classification system, the basic species have unique morphology, can be sexual or apomictic, and are considered as the fundamental evolutionary units or progenitors of the intermediate species. Some basic species can have ancient hybrid/allopolyploid origin (Fehrer et al., 2007a, 2009). By contrast, intermediate species combine morphological characters of two or more basic species and are thought to be derived from hybridizations between basic species. They are usually polyploid and are considered as 'stabilized' taxa because of their predominant apomictic mode of reproduction. They often occupy sizeable areas that are independent of the parental species. Within Pilosella, continuous hybridization also generates recent hybrids that usually co-occur with their parents. They are often represented by only a few individuals within the hybridizing population and produce the highest number of 'variable' progeny, as apomixis appears less penetrant allowing higher frequencies of sexual seed formation (Fehrer et al., 2007b; Krahulec et al., 2008). Within Pilosella, chloroplast DNA (cpDNA) sequences are able to define two distinct and divergent haplotype groups, designated Pilosella I and Pilosella II (Fehrer et al., 2007a; Koltunow et al., 2011).
Genetic and molecular analyses of apomixis in Pilosella have utilized apomicts producing $>93 \%$ apomictic progeny. Two dominant independent loci required for functional apomixis have been identified in R35, a specific isolate of $P$. piloselloides ssp. praealta (formerly treated as Hieracium praealtum) maintained as a vegetative clone by micropropagation (Catanach et al., 2006; Koltunow et al., 2011). The LOSS OF APOMEIOSIS (LOA) locus in R35 is required for apospory, including differentiation of AI cells and suppression of the sexual pathway, while the LOSS OF PARTHENOGENESIS (LOP) locus enables both autonomous embryo and endosperm development. Both loci are transmitted via pollen, and markers linked to both loci have been developed. Markers linked to $L O A$ are non-genic although unique to the $L O A$ region, and both genic and non-genic markers linked to LOP have been developed (Catanach et al., 2006; Koltunow et al., 2011; Okada et al., 2011). The LOA locus is subtelomeric on the long arm of a hemizygous chromosome and is associated with extensive repeats that extend along the long chromosome arm, although these extensive repeats are not essential for $L O A$ function in R35 (Kotani et al., 2014). The chromosomal location of $L O P$ is unknown. Autonomous endosperm formation is controlled by the independent locus $A u t E$ in P. piloselloides isolate D36 (formerly $H$. piloselloides) and is genetically separable from $L O A$ and fertilizationindependent embryogenesis. Markers linked to AutE and its chromosomal location remain to be identified (Ogawa et al., 2013). The genes responsible for apomixis function at all three loci are unknown.

An initial, limited survey in Pilosella identified that the repeat-rich $L O A$-carrying chromosome structure and $L O A$-linked repetitive markers were also conserved in an isolate of $P$. caespitosa (C36; formerly $H$. caespitosum) and in two isolates of $P$. piloselloides (tetraploid D36 and diploid, D18). All examined plants belong to the Pilosella II cpDNA haplotype group. In the R35 and C36 plants, the $L O A$-carrying chromosome is significantly elongated but this is not the case for the D36 and D18 plants (Okada et al., 2011). The elongated $L O A$-carrying chromosome and linked markers were not detected in two apomictic $P$. aurantiaca (formerly $H$. aurantiacum) isolates (A35; A36, both containing Pilosella I cpDNA haplotype) investigated or a sexual $P$. officinarum (formerly $H$. pilosella) isolate (P36). This initial survey therefore revealed a tentative association between apospory-linked $L O A$ marker presence and the Pilosella II cpDNA haplotype (Fehrer et al., 2005, 2007a; Okada et al., 2011).

In this study, we sought to determine whether sexuality also coexists with apomixis in the Hieracium genus, as is the case for Pilosella. We cytologically examined apomixis in 16 Hieracium species. We then utilized a collection of molecular markers linked to apospory (LOA) and autonomous seed development (LOP) in P. piloselloides ssp. praealta to examine the extent of their association with apomixis and its penetrance in Hieracium and Pilosella individuals naturally sourced across Europe. In addition, $L O A$ and $L O P$ marker presence was assessed in experimentally derived progeny obtained from crosses between Pilosella species to study inheritance of the loci. All Pilosella individuals were characterized with respect to their reproductive mode, apomixis penetrance, ploidy level, cpDNA haplotype and species/hybrid origin. This study is one of the first to explore the incidence of apomixis-linked markers in natural populations and has provided insight into the inheritance, penetrance and evolution of apomixis components in Pilosella and Hieracium.

\section{MATERIALS AND METHODS}

\section{Plant material}

Seeds from a total of 20 individuals representing 16 different sexual and apomictic Hieracium species collected in Europe (Table 1) were grown under 
Table 1 Analysis of embryo sac development and apomixis loci-associated molecular marker presence in Hieracium accessions

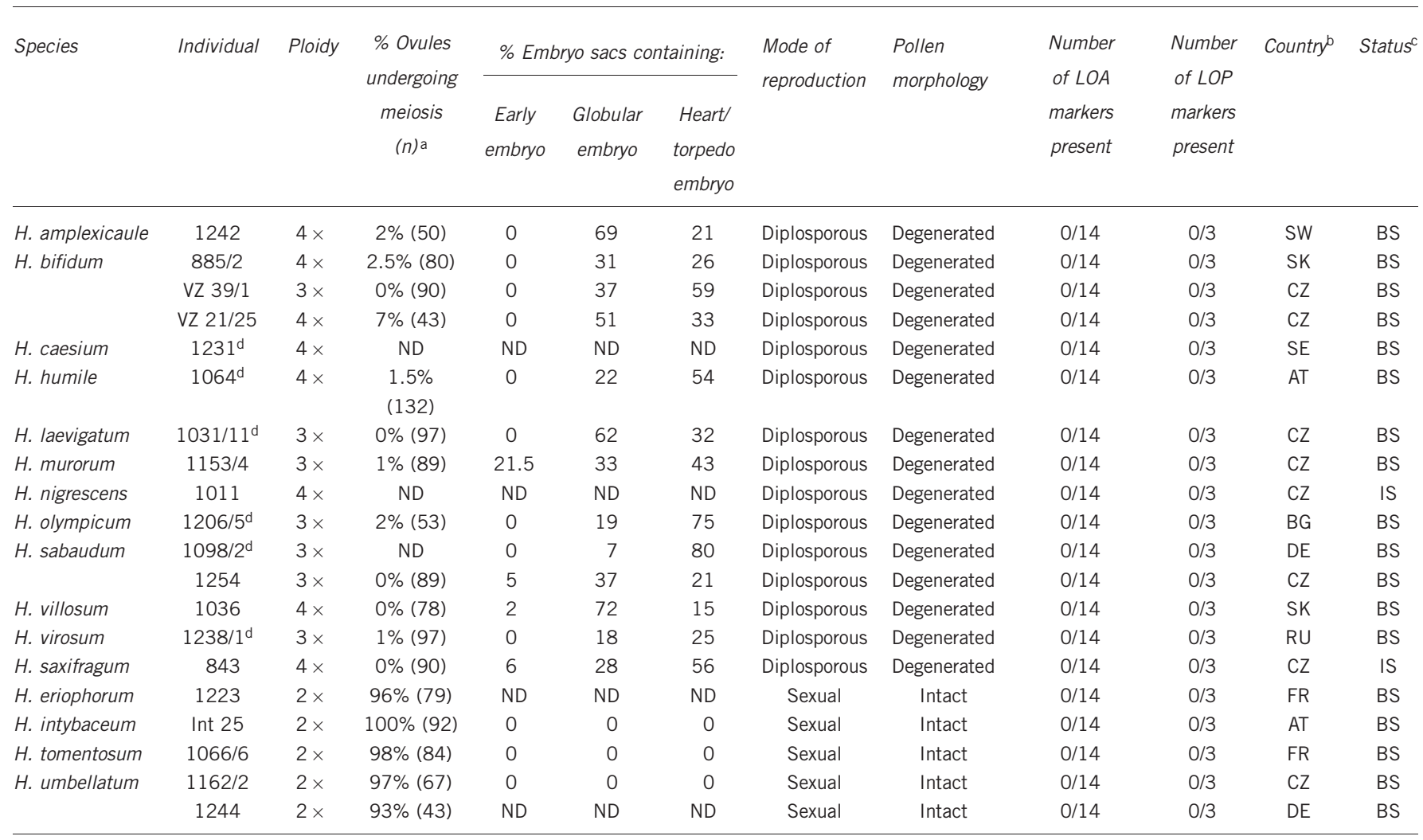

Abbreviations: LOA, LOSS OF APOMEIOSIS; LOP, LOSS OF PARTHENOGENESIS; ND, not determined.

ascored at developmental stage 4 (Koltunow et al., 1998).

bCountry codes: FR, France; CZ, Czech Republic; SK, Slovakia; BG, Bulgaria; DE, Germany; AT, Austria; SW, Switzerland; SE, Sweden; RU, Russia; see also Supplementary Table 1 for more details.

cSpecies status: BS, basic species; IS, intermediate species/stabilized hybridogenous taxon.

${ }^{\mathrm{d}}$ Accession used in Fehrer et al. (2009) and Krak et al. (2013).

quarantine conditions in Australia as described previously for Pilosella (Koltunow et al., 1998). A total of 65 Pilosella plants designated as basic and intermediate species were selected for analysis including recent and experimental hybrids. Species identifications followed the nomenclature of Bräutigam and Greuter (2007) and Bräutigam (2012). The parental origins of the hybrid individuals were inferred based on the recreation of the hybrid phenotype through experimental crosses. Plants were cultivated under identical conditions in the Experimental Garden of the Institute of Botany, Průhonice and details concerning nomenclature of the taxa, geographic origins and voucher information for all plants are provided in Supplementary Table 1.

\section{Cytology, reproduction and penetrance of apomixis}

The DNA ploidy level (relative genome size) of examined plants was estimated from leaf samples by flow cytometry as described in Krahulcová et al. (2004). Capitula of Hieracium plants were staged in terms of morphology as previously described for Pilosella species, and the ovaries were examined at a range of developmental stages as reported previously (Koltunow et al., 1998). Karyotypes in Pilosella were examined using root tip squashes as described by Krahulcová and Krahulec (1999) and chromosome numbers and the presence or absence of an elongated chromosome were scored.

The 65 plants of the Pilosella genus representing basic or intermediate species and recent hybrids were screened for apomixis by decapitation of at least three closed capitula to remove anthers and stigmas before floral opening. Under these conditions apomicts set seed, whereas sexual plants do not (Krahulcová et al., 2004). This test is an approximation for apomictically reproducing plants (Gadella, 1984) because fertilization-independent seed formation is predominantly coupled with apomeiosis (apospory) in wild-type apomicts of Pilosella (Bicknell and Koltunow, 2004). For a more precise determination of the reproductive pathway, the flow cytometric seed screen method was used (Krahulcová et al., 2011). Seeds were harvested from both decapitated capitula (to remove stigma and anthers) and also uncut capitula for comparative seed screening. This method was capable of distinguishing between apomictic plants that had undergone apospory and autonomous seed development $(2 n+0)$ from those which had formed a substantial number of their progeny (embryos) via either haploid parthenogenesis $(n+0$; meiosis with fertilization-independent embryogenesis), the sexual pathway $(n+n)$ or following fertilization of a chromosomally unreduced egg $(2 n+n)$. This enabled an assessment of the penetrance of apospory. Pilosella plants were determined to have a low penetrance of intact, functional apospory when $<75 \%$ of the progeny were of $2 n+0$ origin. Significant efforts were made to obtain multiple apomictic and sexual plant samples per species type. This was not always possible because of the detailed phenotyping and ploidy analyses required for natural accessions over the period of collection.

\section{Screening with $L O A$ and $L O P$-linked apomixis markers}

In total, $14 L O A$ and 3 LOP-associated markers derived from P. piloselloides ssp. praealta $\mathrm{R} 35$ were screened on the Hieracium species, while a smaller set of markers (6 LOA and $1 \mathrm{LOP}$ associated) were used to screen the larger group of Pilosella plants. Marker identity, primer sequences and PCR conditions are specified in Supplementary Table 2. PCR products were scored on $2 \%$ agarose gels as presence or absence of a single strong band of the expected length. Positive and negative controls were included for each PCR (sample R35 and $\mathrm{ddH}_{2} \mathrm{O}$, respectively), and at least two technical repetitions were undertaken per sample. 


\section{CpDNA and LOP haplotype analysis}

The $\operatorname{trn} \mathrm{T}-\operatorname{trn} \mathrm{L}$ intergenic spacer of cpDNA was used to determine the maternal origin of the examined Pilosella plants. Amplification, purification and sequencing of this marker were performed as described in Fehrer et al. (2007a). Assignments of haplotype subgroups, as far as applicable, correspond to Fehrer et al. (2005). The LOP93 marker was examined for its sequence diversity among the different species because of its longer fragment size and genic nature. Sequence editing, alignment and indel coding were performed as described in Fehrer et al. (2007a). Median Networks for trnT-trnL and LOP93 sequences were constructed as implemented in Splitstree 4.11.3 (Huson and Bryant, 2006) with the default settings.

\section{RESULTS}

Apomictic Hieracium species show mitotic diplospory with residual sexuality

Reproductive events were examined in 4 sexual and 12 apomictic Hieracium species (Table 1) by ovule clearing. Figure 1 shows reproductive events in developing unfertilized ovules and anthers of sexual $H$. umbellatum, diplosporous $H$. murorum and, for comparison, aposporous $P$. piloselloides. Events of female gametophyte development in sexual plants were identical to those found in sexual members of Pilosella. A single MMC formed (Figure 1a), underwent meiosis (Figure 1b), megaspore selection and functional megaspore enlargement (Figure 1c), followed by mitotic events to form a 7-celled 8-nucleate embryo sac. Embryo and endosperm formation did not initiate in ovules of unpollinated sexual Hieracium plants. In the diplosporous species examined, the MMC (Figure 1e) did not undergo meiosis in most of the ovules examined, but underwent mitosis to form an embryo sac typical of the events of mitotic diplospory of the Antennaria-type. In 7 of the 15 diplosporous accessions, a small percentage of MMCs (1-7\%) underwent meiosis forming a tetrad of megaspores, demonstrating that diplospory is not obligate (Figure 1f). The remaining MMCs underwent three rounds of mitosis (Figure 1g) and subsequent cellularization and differentiation, forming a mature embryo sac (Figure 1h). Embryo and endosperm formation was fertilization independent in diplosporous embryo sacs and occurred at floret opening in $95 \%$ of cases with the remainder occurring before floret opening.

In aposporous Pilosella species, the MMC progressed through meiosis with AI cells stochastically appearing near cells undergoing meiosis (Figure 1l). The AI cell expanded toward the sexual cells as it underwent mitosis and the sexual pathway ceased. Embryo and endosperm formation was fertilization independent and highly precocious with $35 \%$ of embryos initiating embryogenesis before floret opening (stage 6 of capitulum development; Figure $1 \mathrm{~m}$ ). Sexual Hieracium, sexual Pilosella and apomictic Pilosella accessions produced large quantities of pollen with regular morphology, while pollen formation aborted in most of the diplosporous accessions (Figures $1 \mathrm{n}-\mathrm{p}$ ).

The penetrance of apomixis is variable within facultative Pilosella Sampling of Pilosella was designed to represent a diverse range of accessions within the genus. Analyses of ploidy level and reproductive mode for each of the included individuals confirmed intra-specific diversity for both of these traits (Supplementary Table 3). The basic species sampled generally contained multiple cytotypes (up to four), and most basic species contain both apomictic and sexual individuals. This level of diversity observed in the basic species with regards to ploidy level and reproductive mode, indicates that intra-specific hybridization has been frequent.
Variability in the penetrance of apomixis was also observed. Twelve of the 13 apomictic accessions of basic and intermediate species displayed high penetrance of apomixis producing $>75 \%$ of $2 n+0$ clonal progeny. By contrast, most $2 n+n$ recent hybrids ( 14 out of 15 accessions analyzed) had a lower penetrance of apomixis and thus produced more polyhaploid $(n+0)$ and sexual $(n+n)$ progeny in variable ratios with the frequency of apomictic $(2 n+0)$ progeny being $<75 \%$ of seed set (Supplementary Table 3 ).

Distribution of cpDNA haplotypes throughout Pilosella accessions CpDNA haplotypes were determined for each included Pilosella individual to understand the maternal lineages of the natural and experimental hybrids, and to examine haplotype distributions across different species. Overall, eight distinct haplotype subgroups were observed (Figure 2a), three of them within the previously defined major group Pilosella I, and five within Pilosella II (Fehrer et al., 2005). The majority of the basic species included belonged to the Pilosella II group, with only two basic species attributed to Pilosella I. Individuals of $P$. aurantiaca occurred in both of the haplotype groups; four displayed a Pilosella I haplotype (as was expected based on previous studies), and the remaining two belonged to the Pilosella II cpDNA group. Extensive sharing of cpDNA subtypes among species as well as the occurrence of different subtypes within a species is evident, reflecting their recent speciation and ease with which they can hybridize and introgress.

In those cases when the parental species were sufficiently uniform at the intra-specific level but sufficiently distinct from each other, the maternal progenitor of the intermediate species and recent hybrids could be inferred using the cpDNA sequence (Supplementary Table 3). This was the case for three of the five included intermediate species ( $P$. floribunda, $P$. iserana and $P$. rubra), whose progenitor species belong to different haplotype groups. The maternal progenitor could also be inferred for all of the recent hybrids, with the exception of three $P$. bauhini $\times$ P. officinarum accessions (1433, 1647 and 1015), which have a cpDNA haplotype (II/7 and II/8) found within both progenitor species (Supplementary Table 3).

\section{Extent of conservation of apomixis-linked markers in Hieracium and Pilosella}

Sexual and diplosporous Hieracium species were screened with 14 markers that are non-genic, and specifically linked to the apospory locus $L O A$, in addition to three markers specifically linked to the autonomous seed initiation locus LOP in $P$. piloselloides ssp. praealta (Supplementary Table 2). None of the LOA and LOP markers were detected in the sexual and diplosporous Hieracium species (Table 1).

For Pilosella, a reduced subset of six $L O A$-linked markers was used because of the larger sample size, in addition to the LOP93 genic marker, which is most tightly linked to the LOP locus (Koltunow et al., 2011). The inferred origins of intermediate species and recent hybrids from basic parental species in the Pilosella plants under examination are summarized in Figure 3. Screening of the $L O A$-linked markers revealed the expected marker patterns for the basic species, which have been examined in previous studies (Figure 3). All LOA markers were present as expected in the apomicts $P$. piloselloides ssp. praealta (R35) and $P$. caespitosa, but absent in the apomict $P$. aurantiaca and the sexual cytotypes of $P$. officinarum. Pilosella glacialis, a basic apomictic species collected from France, did not contain any of the tested markers, nor did the apomictic $P$. officinarum individuals. The $L O A$ markers were only present in apomictic basic species of the Pilosella II cpDNA haplotype group, although not all apomictic Pilosella II individuals contained the LOA 

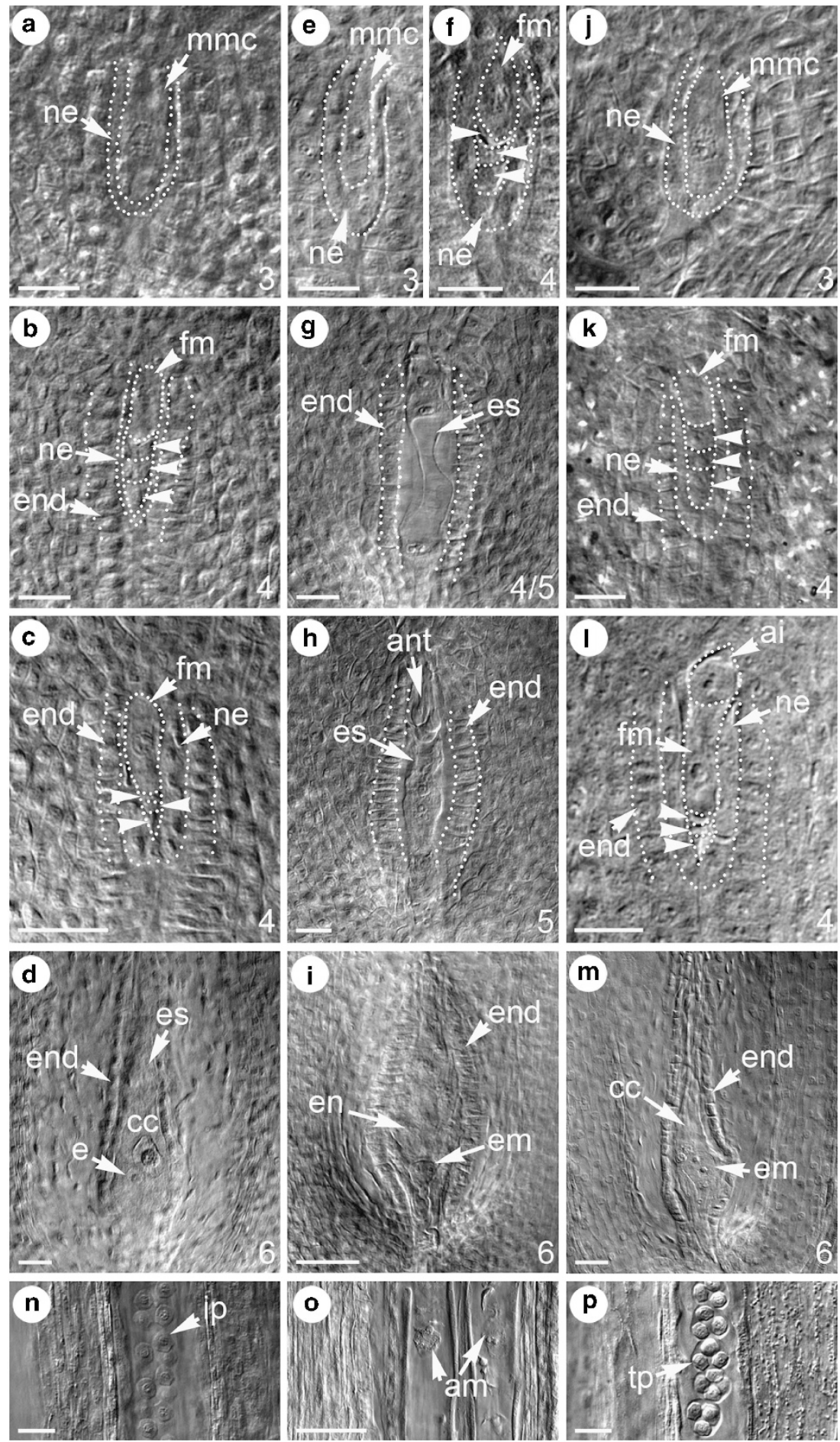

Figure 1 Female gametophyte development in ovules of sexual, diplosporous and aposporous species. Figures a to $m$ show female gametophyte development in ovules of sexual Hieracium umbellatum (a-d), diplosporous Hieracium murorum (e-i) and aposporous Pilosella piloselloides (j-m). Figures $\mathbf{n}$, $\mathbf{o}$ and $\mathbf{p}$ show pollen typical of $H$. umbellatum, H. murorum and P. piloselloides, respectively. Sexual mmc (a) undergoes meiosis to form four megaspores (b), three of which degrade leaving a functional megaspore (c). This functional megaspore undergoes mitosis to form the mature female gametophyte at stage 6 (d). In the diplosporous plant the $\mathrm{mmc}$ (e) usually avoids meiosis and undergoes two mitotic divisions to form a four nucleate embryo sac (g), followed by a further division to form the mature gametophyte (h) by stage 5 . At stage 6 , a precocious embryo and endosperm have formed (i). In some ovules of $H$. murorum, the $\mathrm{mmc}$ follows the sexual pathway and undergoes meiosis to form four megaspores (f). In the aposporous plant, the mmc (j) undergoes meiosis to form four megaspores (k). A cell adjacent to the functional megaspore develops into an aposporous initial (I), which then undergoes mitosis to form the female gametophyte and displaces all the products of meiosis. This has formed a precocious embryo at stage $6(\mathbf{m})$. ai, aposporous initial; am, aborted microspores; ant, antipodal; cc, central cell; e, egg cell; em, embryo; en, endosperm; end, endothelium; es, embryo sac; fm, functional megaspore; ip, immature pollen; mmc, megaspore mother cell; ne, nucellar epidermis; tp, tetrad pollen. Unlabelled arrowheads indicate degrading megaspores. The ovule stage appears in the bottom right of each figure. Scale bars represent $20 \mu \mathrm{m}$ with the exception of sections I, $\mathbf{m}$ and $\mathbf{0}$, which correspond to $50 \mu \mathrm{m}$. 
a

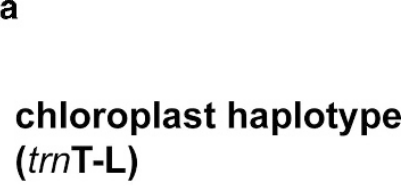

\begin{tabular}{|c|c|c|c|}
\hline $\begin{array}{l}\text { 1732(R35) P. praealta } \\
1164 \text { P. bauhini }\end{array}$ & $\begin{array}{l}1297 \text { P. officinarum } \\
1440 P \text {. officinarum }\end{array}$ & $\begin{array}{l}\text { NZGO432 P. officinarum } \\
\text { NZ PP PI6 P. officinarum }\end{array}$ & $\begin{array}{l}1980 \text { P. glomerata } \\
1605 \text { P. caespitosa }\end{array}$ \\
\hline 1645 P. bauhini & $1113 P$. officinarum & $74 P$. officinarum & 1312 P. caespitosa \\
\hline 1921 P. bauhini & $1660 P$. officinarum & $1417 P$. officinarum & 1315 cae $\times$ aur \\
\hline $\begin{array}{l}1920 \text { P. bauhini } \\
1545 \text { P. bauhini }\end{array}$ & $1548 P$. officinarum & $1505 P$. officinarum & $1326 P$ aurantiaca \\
\hline 1433 bau $x$ off & & & \\
\hline 1647 bau $\times$ off & & II/7 & \\
\hline
\end{tabular}

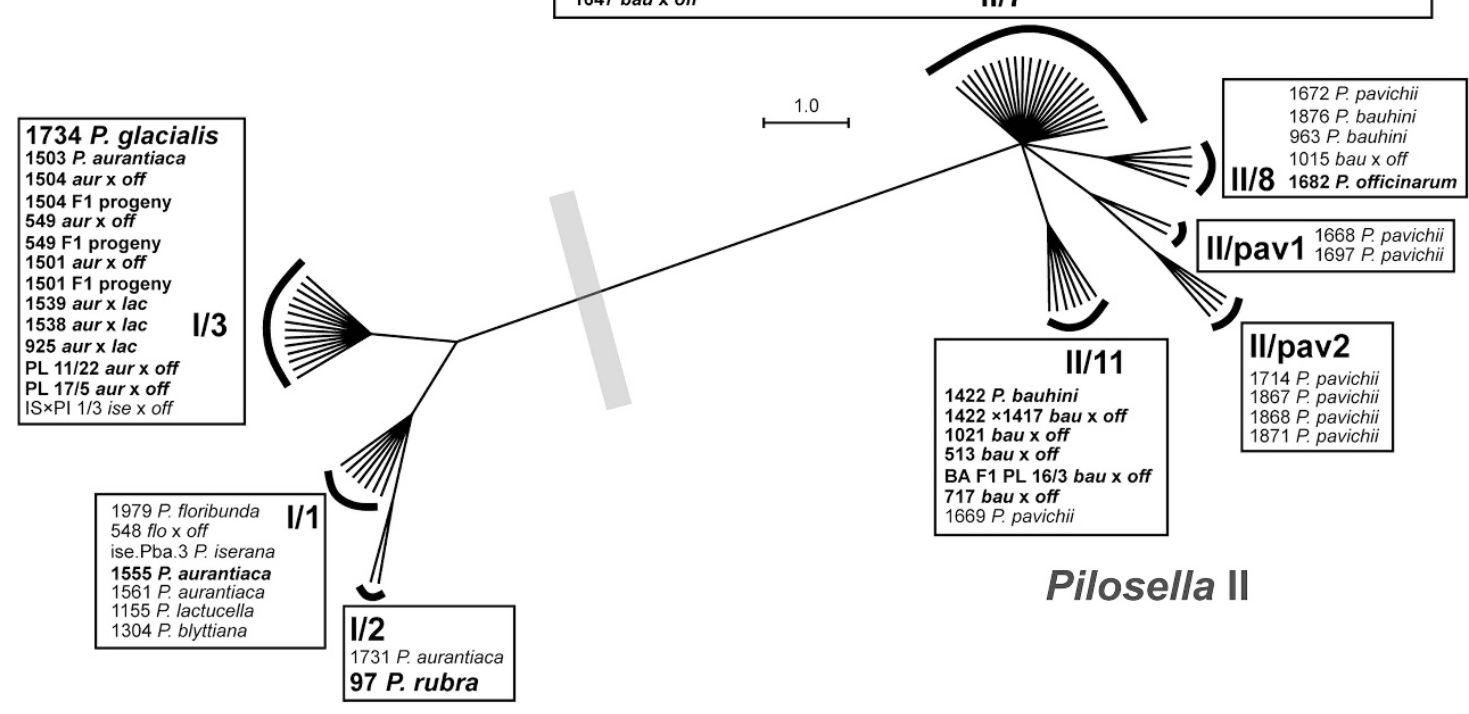

Pilosella I

b

\section{LOP-associated marker}

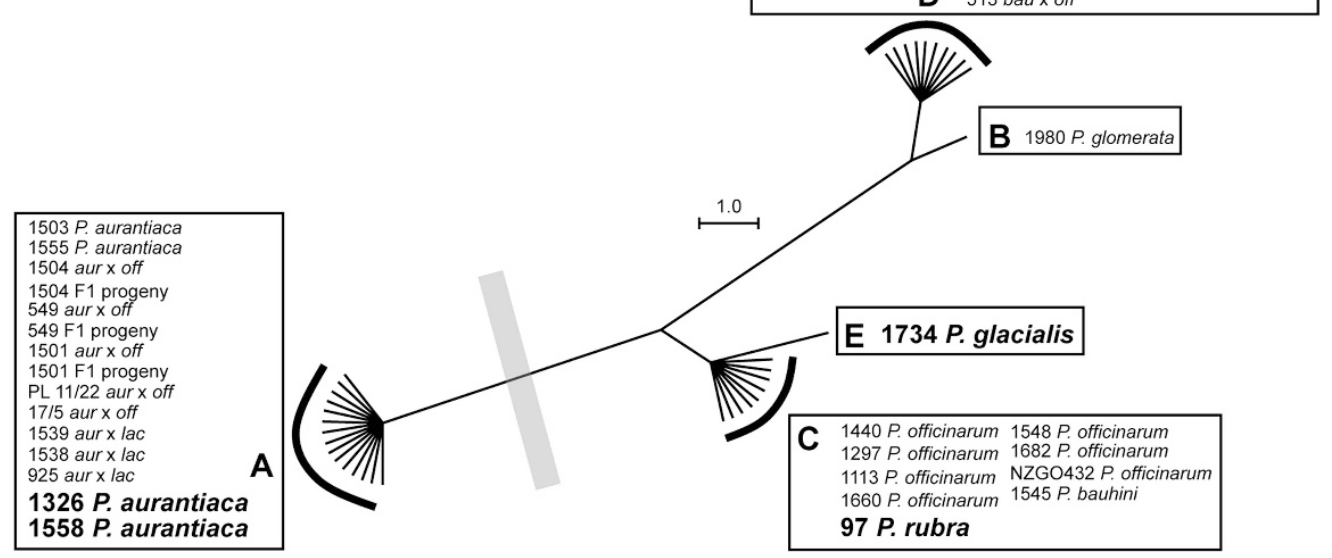

Figure 2 Median networks of chloroplast haplotype (a) and LOP93 marker (b) sequences. (a) The major cpDNA haplotype groups Pilosella I and Pilosella II are shown along with an assignment of individual plants to subtypes within each group according to Fehrer et al. (2005) (see also Supplementary Table 3). Those that are LOP93 positive are indicated in bold. (b) Five haplotypes (A-E) of the LOP-associated marker can be distinguished. (a, b) The grey bar indicates a general correspondence of taxa possessing the major cpDNA haplotype group Pilosella I and LOP93 haplotype A versus Pilosella II and LOP93 B-E. Four individuals not matching this pattern ( $P$. glacialis 1734, $P$. aurantiaca 1326 and 1558, P. rubra 97) are shown in bold and larger font in both networks. They indicate transmission of $L O P$ via pollen.

markers (Figure 3). No correlation between $L O A$ marker presence and cpDNA haplotype was therefore observed.

LOA-linked markers were absent in sexual Pilosella plants, with the exception of two individuals that possessed a single marker (14-T7, Supplementary Table 3). These two individuals belong to the P. piloselloides aggregate species $P$. bauhini and P. pavichii. LOA marker presence in these two species is variable and may indicate that recombination at the $L O A$ locus has accompanied intra-specific hybridization. Apomictic P. bauhini individuals have either all, some (one to three) or none of the $L O A$ markers. Similarly, all apomictic $P$. pavichii individuals contained only two to four $L O A$ markers, with an inconsistent pattern observed between the individuals (Supplementary Table 3).

An elongated chromosome carries the $L O A$ locus in P. caespitosa and P. piloselloides ssp. praealta (Okada et al., 2011). Karyotyping revealed that an elongated chromosome was present in four out of seven of the apomictic basic species, all of which belong to Pilosella II (Figure 3). The elongated chromosome can be stably transmitted 


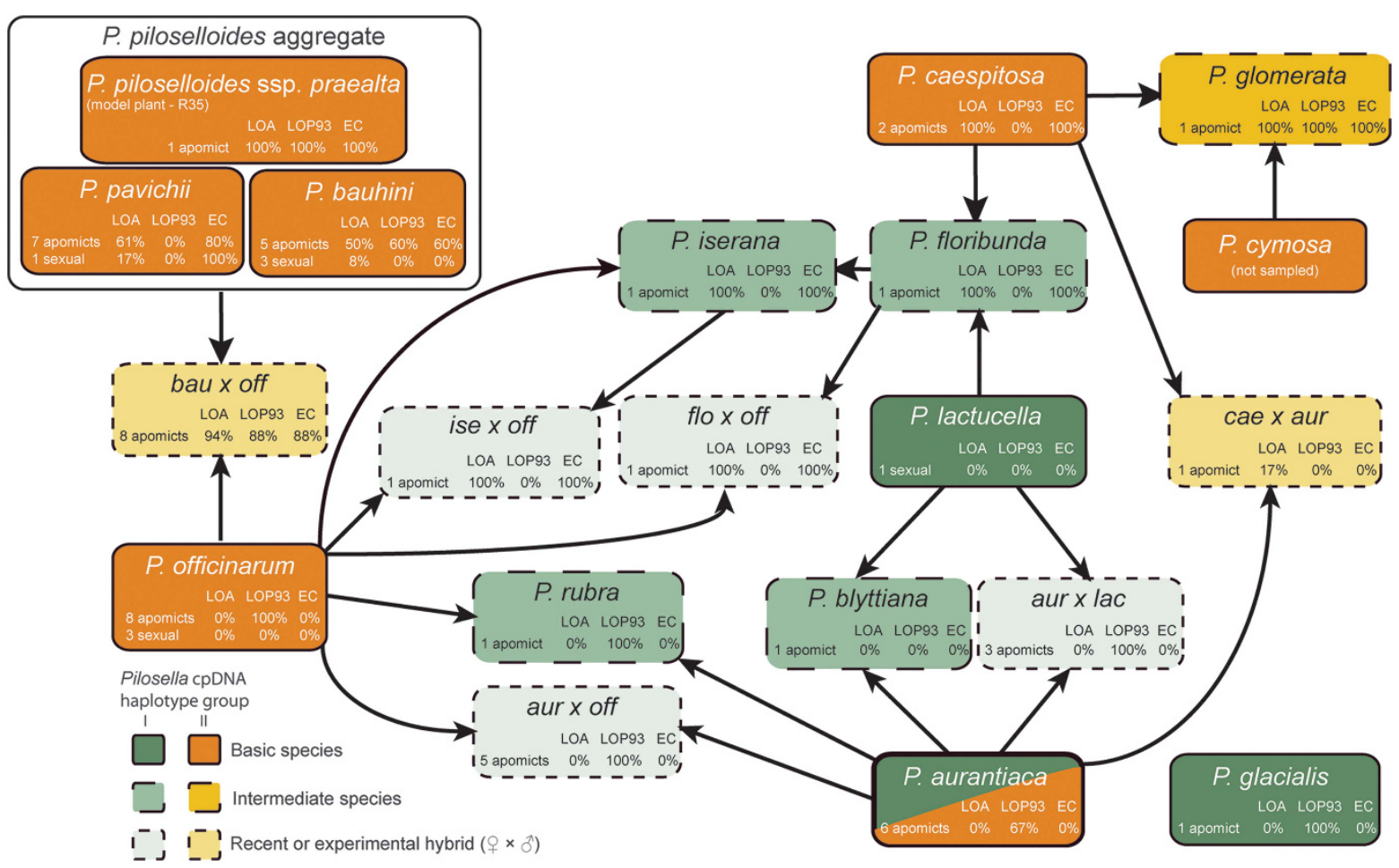

Figure 3 Summary of Pilosella species, cpDNA haplotype group, reproduction and apomixis-associated markers. This diagram depicts the included Pilosella species. Putative hybridization events between various basic species that gave rise to intermediate species and phenotypically corresponding recent hybrids are represented by arrows. The major chloroplast haplotype group for a given species is represented by either green (Pilosella I) or orange (Pilosella II) shading. If both haplotype groups were identified within a species, they are shaded with both colours. For each taxon, the number of individuals sampled is given along with their reproductive mode, the conservation of $L O A$ and $L O P$-associated markers and the occurrence of the hemizygous elongated chromosome (EC). For $L O A$-associated markers, markers within the core region were included and calculated as a weighted average of each taxon. Detailed information for each individual including ploidy, cpDNA subtype, particular $L O A$-associated markers, $L O P$ haplotype, penetrance of apomixis and geographic origin is given in Supplementary Table 3. For explanations concerning the P. piloselloides species aggregate, see Supplementary Table 1.

within Pilosella following hybridization because intermediate species, as well as recent and experimental hybrids can inherit the elongated chromosome along with the apomixis phenotype, when derived from an elongated chromosome-carrying progenitor species (Figure 3). Transmission of the elongated chromosome occurs in $2 n+n$ hybrid progeny as maternal meiosis is absent, thus the entire unreduced $(2 n)$ maternal genome is inherited together with paternal chromosomes in nuclei following fertilization. Using this knowledge in conjunction with karyotype patterns, $P$. bauhini can be inferred as the maternal progenitor of two P. bauhini $\times$ P. officinarum hybrids $(1433,1647)$ for which maternal determination was inconclusive using cpDNA. Within P. bauhini and P. pavichii, not all apomictic individuals contained the long chromosome (Supplementary Table 3). This is indicative of potential recombination and coincident loss of elongated chromosome integrity as observed in rare recombinants in experimental cross populations (Kotani et al., 2014).

Within Pilosella, the LOP93 marker was absent in all of the sexually reproducing individuals. Only $60 \%$ of the apomicts possessed the LOP93 marker indicating that it does not show perfect correlation with autonomous seed development (Supplementary Table 3). LOP93 was present in five of the seven apomictic basic species included in the study. An association of LOP93 with LOA markers was not always found. For example, $P$. caespitosa had all LOA markers, but lacked LOP93 and while no $P$. aurantiaca individuals carried LOA markers, four out of six had LOP93 (Figure 3).

\section{Inheritance of $L O A$ and $L O P$-linked markers in Pilosella}

The inheritance of $L O A$-linked markers could also be studied using the naturally occurring stabilized intermediate species and the recent and experimental hybrids (Figure 3). Apomictic intermediate species inherited the same $L O A$ genotype as their apomictic progenitor; that is, $P$. caespitosa intermediate species ( $P$. floribunda, $P$. glomerata and $P$. iserana) were $L O A$ positive, whereas $P$. aurantiaca intermediate species ( $P$. blyttiana and $P$. rubra) were $L O A$ negative. This was also the case for the recent hybrids, including those with low penetrance of apomixis. No difference in $L O A$-associated marker pattern was therefore observed between plants with high and low levels of penetrance. In the single sample that is inferred to be a recent hybrid of two apomicts ( $P$. caespitosa ( $L O A$ positive) $\times P$. aurantiaca ( $L O A$ negative)), only one of the $L O A$-linked markers was present. In this instance, the apospory locus has probably been inherited from P. aurantiaca.

When present, the LOP93 amplicon was sequenced to investigate the level of nucleotide diversity and possible evolutionary relationships within Pilosella. Sequencing revealed five different haplotypes (Figure $2 \mathrm{~b}$ ), which were distinguished by 13 substitutions and one 1-bp indel. For each sample sequenced, no variation was observed within the individual, as all reads provided a clear signal and no visible single-nucleotide polymorphisms in direct sequencing. In general, a single haplotype was associated with each species group, with the exception of $P$. bauhini ssp. bauhini for which two different haplotypes ( $\mathrm{C}$ and $\mathrm{D}$ ) were observed from the two individuals tested. 
This species specificity is notable given the frequent hybridization known to occur within Pilosella. Analysis of the LOP93 haplotype relationships showed a strong correlation with the cpDNA haplotypes (Figure 2b), where the LOP93 A haplotype was found in Pilosella I individuals, and LOP93 haplotypes B-E were restricted to Pilosella II. Exceptions to this association include $P$. rubra (97) and $P$. glacialis (1734), which contained Pilosella I cpDNA and LOP93 haplotype C. Also, the two $P$. aurantiaca individuals (1326 and 1558), which contained unexpected Pilosella II cpDNA do not fit the correlation as they had LOP93 A haplotypes.

\section{DISCUSSION}

\section{Residual sexuality within apomictic Hieracium}

Hieracium and Pilosella genera are very closely related, yet are characterized by different mechanisms of gametophytic apomixis, diplospory and apospory respectively. Early cytological studies in Hieracium focused on a few species identifying apomicts as diplosporous often with non-viable pollen resulting from abnormal pollen meiosis (Rosenberg, 1927; Gentcheff and Gustafsson, 1940; Bergman, 1941; Skawińska, 1963). Diplosporous Hieracium species have been widely regarded as obligate apomicts and the genetic inheritance of apomixis is unknown in Hieracium. All apomictic plants identified here displayed mitotic diplospory of the Antennaria type.

We observed meiosis and tetrad formation in $1-7 \%$ of ovules in seven of the apomicts (Table 1). Skawińska (1963) also observed meiotic tetrads, which subsequently aborted in Hieracium alpinum. These observations of trace capacity for sexual female gamete formation in these diplosporous Hieracium are consistent with a potential facultative reproductive nature; however, it is unclear if viable chromosomally reduced eggs are produced in these plants. If so, it would be expected that a small fraction of polyhaploid $(n+0)$ progeny could form from the parthenogenetic development of a reduced egg cell, as is known to occur in Pilosella (Bicknell et al., 2003; Krahulcová et al., 2004). However, as the majority of the Hieracium species are triploid (in contrast to Pilosella), embryo development and seedling survival would depend upon egg viability following meiosis. Further analyses such as large-scale flow cytometric seed screens of diplosporous species (Matzk et al., 2000) are needed to examine this possibility.

Assumed obligate apomixis in Hieracium has been proposed as a contributing factor in the lack of recent hybridization seen within this genus, and subsequently as one of the key reasons for population structure differences between Hieracium and the facultative Pilosella (Mráz et al., 2011). Given the potential for residual sexuality of Hieracium suggested in this study, the population structure of Hieracium is possibly best explained by a combination of factors including reduced gene flow due to limited pollen viability in some species, self-fertilization following breakdown in self-incompatibility (Mráz, 2003) and precocious embryony, whereby some embryo formation initiates before anthesis.

\section{Apospory in Pilosella and diplospory in Hieracium have probably evolved independently}

The development of markers physically linked to the $L O A$ and $L O P$ loci of $P$. piloselloides ssp. praealta has provided the opportunity to assess their conservation throughout a larger sample of naturally sourced Hieracium and Pilosella species. The absence of Piloselladerived apomixis-linked markers in Hieracium species may be an indication that apospory and diplospory have evolved independently in Pilosella and Hieracium, respectively. Based on phylogenetic distributions across angiosperm species, apomixis is thought to have evolved independently many times including multiple times within some genera (Carman, 1997; Hörandl and Hojsgaard, 2012). Carman (1997) documented 19 genera where both diplospory and apospory are evident. Our findings are therefore consistent with the polyphyletic description of apomixis. The lack of apospory markers within diplosporous Hieracium also agrees with the generally accepted idea of apospory and diplospory being non-homologous, given the different developmental pathways that define them (Van Dijk and Vijverberg, 2005). As both Hieracium and Pilosella have the capacity to undergo autonomous seed development, absence of LOP-associated markers in Hieracium species suggests that this process may also have evolved independently in Hieracium and Pilosella species.

The possibility remains, however, that Hieracium species do possess the same apomixis loci as Pilosella but they are undetectable using the markers used in this study, as they are not causal for apomixis. Genome divergence between Hieracium and Pilosella may also have interfered with cross-species marker amplification. To conclusively investigate whether the Pilosella-derived apomixis-linked sequences are present or rearranged within the genome of Hieracium species, future experiments could involve DNA hybridization of current linked sequences or amplification of genic sequences responsible for both apospory and autonomous seed formation following their identification within Pilosella.

\section{Patterns of conservation and inheritance of $L O A$ and $L O P$-linked markers in Pilosella}

LOA-linked markers were absent in all but two sexual Pilosella accessions that each contained only one marker, while the $L O A$ markers predominated in basic species of the Pilosella II cpDNA haplotype group. This supported a strong association with apospory as indicated by the prior limited survey (Okada et al., 2011). Absence of $L O A$-linked markers in some apomictic basic species, and their corresponding stabilized and recent hybrids also substantiate previous work and may be indicative of the markers being non-genic and imperfect. Given the facultative nature of apomixis in Pilosella, another possibility is that the apomictic $L O A$ marker negative species observed here have lost the $L O A$ flanking marker sequences through recombination. The sporadic $L O A$ marker patterns observed in the basic Pilosella species $P$. bauhini and $P$. pavichii, for example, could be interpreted as evidence of recombination at the $L O A$ locus or alternatively as a series of fixed point mutations preventing marker amplification. Further analysis of more individuals per species will help clarify whether marker absence is due to meiotic recombination.

It is also possible that the $L O A$-linked marker negative taxa, such as apomictic $P$. aurantiaca, $P$. glacialis and $P$. officinarum, may represent lineages where apospory is caused by another genomic region distinct in function from LOA. This would imply that an alternate apospory pathway may have evolved within Pilosella that is responsible for regulating apospory in these $L O A$-negative species. The notion of multiple apospory pathways within Pilosella may be supported by the observation of subtly different phenotypes when apospory is studied at the cytological level. P. piloselloides (LOA marker positive) and $P$. aurantiaca ( $L O A$ marker negative) differ with respect to the timing and mode of aposporous embryo sac formation (Koltunow et al., 2000, 2011). Furthermore, experimental P. piloselloides $\times$ $P$. aurantiaca hybrids display a range of developmental alterations in apospory compared with the parental species (Koltunow et al., 2000). The developmental alterations seen in these hybrids may possibly be due to the co-expression of different apospory-inducing genes or pathways that exist in the parental species. Apospory appears to have arisen independently at least three times in Ranunculus (Van 
Dijk and Vijverberg, 2005). Thus, the same may have occurred in Pilosella.

As with apospory-associated markers, the genic LOP93 marker also showed variable conservation throughout Pilosella (Supplementary Table 3), which demonstrated that it is also a useful, but not perfect marker for autonomous seed development. Absence of the marker may be the result of the range of possibilities already discussed above for the LOA markers. Alternatively, the LOP locus may genuinely be absent, which may suggest different routes to autonomous seed development within Pilosella. In any case, the lack of a strict association between $L O A$ and $L O P$ markers throughout the population is interesting and supports the proposition that apospory and autonomous seed formation are independently inherited and evolved as separate traits.

Sequencing of the LOP93 amplicon, when present, provided the opportunity to examine how this locus is inherited and also how it has evolved within Pilosella. LOP93 sequence revealed intriguing findings, including low sequence divergence across species, only a single haplotype per species (irrespective of ploidy level) and species specificity of the $L O P$ haplotypes. As a result of the ease of hybridization between Pilosella species and cytotypes, species-specific markers are thought to be rare. Certainly, cpDNA is not strictly species specific (Fehrer et al., 2007a). The LOP93 haplotypes, however, show almost complete species specificity for the accessions analyzed (Figure $2 \mathrm{~b}$ ).

Furthermore, the association seen between the LOP93 and cpDNA haplotypes indicates that $L O P$ is often transmitted maternally. The exceptions to this trend include four individuals, which have apparently inherited $L O P$ via pollen. Thus, comparison of LOP93 and cpDNA haplotypes revealed in many cases the parent from which $L O P$ was obtained, confirming that it can be inherited both maternally or paternally in natural populations. LOP confers the essential capability to form a viable seed independent of the mode of female gametophyte formation and ploidy of the egg (that is, $n$ or $2 n$ ). Therefore, LOP transmissibility via male and female gametes maximizes seed formation and fecundity in populations. It would be interesting to determine if there is a stronger selection pressure for $L O P$ in apomictic populations. As chromosome walking continues along the $L O A$ and $L O P$ loci, the development of perfect causal gene markers will help clarify this and determine the degree of conservation of apomixis-inducing pathways within Pilosella.

\section{Paternal modifiers influence expressivity of apomixis in $2 n+n$ Pilosella}

We have discovered that at least three loci are required for the qualitative traits of apomixis in Pilosella to confer apospory and fertilization-independent embryo and endosperm formation. Analyses of crosses between sexual and apomictic species demonstrate the variable penetrance of apomixis in progeny suggesting that other, quantitative factors or modifiers may influence the final reproductive behaviour in hybrids (Koltunow et al., 2000; Krahulcová et al., 2011; Tucker et al., 2012). However, to date, apomixis penetrance has not been correlated to the presence of the $L O A$ and $L O P$ loci.

Low penetrance or expressivity of apomixis has been previously reported within naturally sourced and experimental $2 n+n$ hybrids arising from crosses between a range of Pilosella apomicts as the maternal parent ( $P$. bauhini, P. floribunda and P. aurantiaca) and sexual $P$. officinarum as the pollen donor (Krahulec et al., 2008; Krahulcová et al., 2009; Krahulcová et al., 2011). These hybrids arise from the fertilization of a chromosomally unreduced egg. This study analyzed a greater range of $2 n+n$ hybrids from crosses between apomicts ( $P$. bauhini, P. floribunda, $P$. iserana and $P$. aurantiaca) and sexual $P$. officinarum, all of which (except one) displayed low penetrance of apospory (Supplementary Table 3). Each $2 n+n$ hybrid contained the same $L O A$ marker patterns as their maternal apomictic parent confirming inheritance of the primary apospory locus in the inherited maternal genome. The low expressivity of apomixis in such hybrids indicates that the penetrance of apomixis can be affected by parental genome interactions following hybridization. These may involve factors such as modifiers, dosage effects and epistatic interactions arising from the incoming paternal genome. Interestingly, in polyhaploid progeny $(n+0)$ arising from multiple $2 n+n$ $P$. aurantiaca $\times P$. officinarum plants, apomixis was highly penetrant (Supplementary Table 3). This suggests that the suppressive modifiers may have been lost following meiotic reduction, or genome dosage and epistatic factors have been removed. Recovery of apomixis penetrance via a meiotic event clearly suggests that these modifiers are unlinked and can be lost over various meiotic cycles. Future cytological screening of those plants with low expressivity of apomixis will help to determine exactly which reproductive processes are being affected.

\section{Conclusions and future directions}

This study demonstrated that meiosis can occur at low levels in diplosporous Hieracium, indicating the potential for progeny to arise via the sexual pathway. The absence of the $L O A$ and $L O P$-linked markers developed from aposporous Pilosella species in the examined diplosporous Hieracium plants tested, suggests independent evolution of apomixis in these two genera, although further analyses with perfect, causal gene markers would be required to conclusively determine this. Within Pilosella, LOA and LOP markers were essentially absent within the sexual plants, and conserved in many but not all apomictic species, which may imply multiple origins of apomixis even within Pilosella. Marker presence also had no association with the penetrance of apospory, and overall, many associations tested relating to phenotype and genotype were only weakly correlated, which indicates a complex evolutionary history. Evidence for independence of $L O A$ and $L O P$ loci was suggested in natural populations, which supports previous experimental observations. To further understand the evolution of apomixis loci within Pilosella, perfect markers for the causal genes at $L O A$ and $L O P$ loci are actively being sought to screen across natural populations. A molecular phylogeny of apomictic Pilosella species based on neutral nuclear markers is still lacking, mostly because of complications due to the high ploidy of most apomicts $(4 \times-7 \times)$ and their almost unlimited potential to hybridize. Knowledge of species relationships within Pilosella would contribute to the understanding of apomixis evolution within this complex genus.

\section{DATA ARCHIVING}

Sequence data have been submitted to GenBank: accession numbers KF196158-KF196254. Voucher specimens of plants analyzed are deposited in the Herbarium PRA (Průhonice, Czech Republic).

\section{CONFLICT OF INTEREST}

The authors declare no conflict of interest.

\section{ACKNOWLEDGEMENTS}

We thank Siegfried Bräutigam for expert determinations of the plant material and help with the taxonomic background for this paper. This research was supported by a Science and Industry Endowment Fund grant to AMGK and funding from the Czech Science Foundation (206/08/0890, P506/10/1363) and the Academy of Sciences of the Czech Republic (RVO 67985939). 
Bergman B (1941). Studies on the embryo sac mother cell and its development in Hieracium subg. Archieracium. Svensk Bot Tidskr 35: 1-41.

Bicknell RA, Koltunow AM (2004). Understanding apomixis: recent advances and remaining conundrums. Plant Cell 16: S228-S245.

Bicknell RA, Lambie SC, Butler RC (2003). Quantification of progeny classes in two facultatively apomictic accessions of Hieracium. Hereditas 138: 11-20.

Bräutigam S (2012). Pilosella. In: Greuter W, Raus T (eds) Med-Checklist Notulae, 31. Willdenowia. Vol 42, pp 290.

Bräutigam S, Greuter W (2007). A new treatment of Pilosella for the Euro-Mediterranean flora. Willdenowia 37: 123-137.

Carman JG (1997). Asynchronous expression of duplicate genes in angiosperms may cause apomixis, bispory, tetraspory, and polyembryony. Biol J Linn Soc 61: 51-94.

Catanach AS, Erasmuson SK, Podivinsky E, Jordan BR, Bicknell R (2006). Deletion mapping of genetic regions associated with apomixis in Hieracium. Proc Natl Acad Sci USA 103: 18650-18655.

Fehrer J, Gemeinholzer B, Chrtek Jr J, Bräutigam S (2007a). Incongruent plastid and nuclear DNA phylogenies reveal ancient intergeneric hybridization in Pilosella hawk weeds (Hieracium, Cichorieae, Asteraceae). Mol Phylogenet Evol 42: 347-361.

Fehrer J, Krahulcová A, Krahulec F, Chrtek Jr J, Rosenbaumová R, Bräutigam S (2007b) Evolutionary aspects in Hieracium subgenus Pilosella. In: Hörandl E, Grossniklaus U, van Dijk P, Sharbel T (eds) Apomixis: Evolution, Mechanisms and Perspectives. Koeltz: Königstein, pp 359-390.

Fehrer J, Krak K, Chrtek J (2009). Intra-individual polymorphism in diploid and apomictic polyploid hawkweeds (Hieracium, Lactuceae, Asteraceae): disentangling phylogenetic signal, reticulation, and noise. BMC Evol Biol 9: 239

Fehrer J, Šimek R, Krahulcová A, Krahulec F, Chrtek Jr J, Bräutigam S (2005). Evolution, hybridisation, and clonal distribution of apo- and amphimictic species of Hieracium subgen. Pilosella (Asteraceae, Lactuceae) in a Central European mountain range. In: Bakker F, Chatrou L, Gravendeel B, Pelser P (eds) Plant Species-Level Systematics: New Perspectives on Pattern \& Process. Regnum Vegetabile. A. R. G. Gantner Verlag:: Rugell. vol 143, pp 175-201.

Gadella TWJ (1984). Cytology and the mode of reproduction of some taxa of Hieracium subgenus Pilosella. Proc Kon Ned Acad Wetensch 87: 387-399.

Gentcheff G, Gustafsson A (1940). The balance system of meiosis in Hieracium. Hereditas 26: 209-249.

Harlan JR, deWet JMJ (1975). On Ö. Winge and a prayer: the origins of polyploidy. Bot Rev 41: 361-390

Hörandl E, Hojsgaard D (2012). The evolution of apomixis in angiosperms: a reappraisal. Plant Biosyst 146: 681-693.

Huson DH, Bryant D (2006). Application of phylogenetic networks in evolutionary studies. Mol Biol Evol 23: 254-267.

Koltunow AM, Johnson SD, Bicknell RA (1998). Sexual and apomictic development in Hieracium. Sex Plant Reprod 11: 213-230.

Koltunow AM, Johnson SD, Bicknell RA (2000). Apomixis is not developmentally conserved in related, genetically characterized Hieracium plants of varying ploidy. Sex Plant Reprod 12: 253-266.

Koltunow AMG, Johnson SD, Rodrigues JCM, Okada T, Hu Y, Tsuchiya T et al. (2011). Sexual reproduction is the default mode in apomictic Hieracium subgenus Pilosella, in which two dominant loci function to enable apomixis. Plant J 66: 890-902.

Koltunow AM, Ozias-Akins P, Siddiqi I (2013). Apomixis. In: Becraft PW (ed.) Seed Genomics. John Wiley \& Sons, pp 83-110.

Kotani Y, Henderson ST, Suzuki G, Johnson SD, Okada T, Siddons H et al. (2014). The LOSS OF APOMEIOSIS ( $L O A$ ) locus in Hieracium praealtum can function independently of the associated large-scale repetitive chromosomal structure. New Phytol 201: 973-981.
Krahulcová A, Krahulec F (1999). Chromosome numbers and reproductive systems in selected representatives of Hieracium subgen. Pilosella in the Krkonoše Mts (the Sudeten Mts). Preslia 71: 217-234.

Krahulcová A, Krahulec F, Rosenbaumová R (2011). Expressivity of apomixis in $2 n+n$ hybrids from an apomictic and a sexual parent: insights into variation detected in Pilosella (Asteraceae: Lactuceae). Sex Plant Reprod 24: 63-74.

Krahulcová A, Papoušková S, Krahulec F (2004). Reproduction mode in the allopolyploid facultatively apomictic hawkweed Hieracium rubrum (Asteraceae, H. subgen. Pilosella). Hereditas 141: 19-30.

Krahulcová A, Rotreklová O, Krahulec F, Rosenbaumová R, Plačková I (2009). Enriching ploidy level diversity: the role of apomictic and sexual biotypes of Hieracium subgen. Pilosella (Asteraceae) that coexist in polyploid populations. Folia Geobot 44: 281-306.

Krahulec F, Krahulcová A, Fehrer J, Bräutigam S, Schuhwerk F (2008). The structure of the agamic complex of Hieracium subgen. Pilosella in the Sumava Mts and its comparison with other regions in Central Europe. Preslia 80: 1-26.

Krak K, Caklová P, Chrtek J, Fehrer J (2013). Reconstruction of phylogenetic relationships in a highly reticulate group with deep coalescence and recent speciation (Hieracium, Asteraceae). Heredity 110: 138-151.

Matzk F, Meister A, Schubert I (2000). An efficient screen for reproductive pathways using mature seeds of monocots and dicots. Plant J 21: 97-108.

Mráz P (2003). Mentor effects in the genus Hieracium s.str. (Compositae, Lactuceae). Folia Geobot 38: 345-350.

Mráz P, Chrtek J, Fehrer J (2011). Interspecific hybridization in the genus Hieracium s. str.: evidence for bidirectional gene flow and spontaneous allopolyploidization. Plant Syst Evol 293: 237-245

Mráz P, Chrtek J, Fehrer J, Plačková I (2005). Rare recent natural hybridization in Hieracium s. str.-evidence from morphology, allozymes and chloroplast DNA. Plant Syst Evol 255: 177-192.

Mráz P, Paule J (2006). Experimental hybridization in the genus Hieracium s. str. (Asteraceae): crosses between selected diploid taxa. Preslia 78: 1-26.

Nägeli C, Peter A (1885). Die Hieracien Mittel-Europas, Piloselloiden. R. Oldenbourg: München

Ogawa D, Johnson SD, Henderson ST, Koltunow AM (2013). Genetic separation of autonomous endosperm formation (AutE) from two other components of apomixis in Hieracium. Plant Reprod 26: 113-123.

Okada T, Ito K, Johnson SD, Oelkers K, Suzuki G, Houben A et al. (2011). Chromosomes carrying meiotic avoidance loci in three apomictic eudicot Hieracium subgenus Pilosella species share structural features with two monocot apomicts. Plant Physiol 157: $1327-1341$.

Ozias-Akins P (2006). Apomixis: developmental characteristics and genetics. Crit Rev Plant Sci 25: 199-214.

Rosenberg 0 (1927). Die semiheterotypische Teilung und ihre Bedeutung für die Entstehung verdoppelter Chromosomenzahlen. Hereditas 8: 305-338.

Skawińska R (1963). Apomixis in Hieracium alpinum L. Acta Biol Cracov 5: 7-14.

Slade K, Rich TCG (2007). Pollen studies in British Hieracium sect. Alpina (Asteraceae). Watsonia 26: 443-450.

Tucker MR, Koltunow AMG (2009). Sexual and asexual (apomictic) seed development in flowering plants: molecular, morphological and evolutionary relationships. Funct Plant Biol 36: 490-504.

Tucker MR, Okada T, Johnson SD, Takaiwa F, Koltunow AMG (2012). Sporophytic ovule tissues modulate the initiation and progression of apomixis in Hieracium. J Exp Bot 63 3229-3241.

Van Dijk P, Vijverberg K (2005). The significance of apomixis in the evolution of the angiosperms: a reappraisal. In: Bakker F, Chatrou L, Gravendeel B, Pelser P (eds) Plant Species-Level Systematics: New Perspectives on Pattern and Process. Gantner Verlag: Ruggell, Liechtenstein, pp 101-116.

Zahn KH (1921-1923). Hieracium. In: Engler A (ed.) Das Pflanzenreich. Wilhelm Engelmann: Leipzig. Vol 4.

Supplementary Information accompanies this paper on Heredity website (http://www.nature.com/hdy) 